\title{
A Recursive Scheme of First Integrals of the Geodesic Flow of a Finsler Manifold ${ }^{\star}$
}

Willy SARLET

Department of Mathematical Physics and Astronomy, Ghent University, Krijgslaan 281, B-9000 Ghent, Belgium

E-mail: willy.sarlet@ugent.be

URL: http://maphyast.ugent.be

Received October 30, 2006, in final form January 17, 2007; Published online February 13, 2007

Original article is available at http://www.emis.de/journals/SIGMA/2007/024/

\begin{abstract}
We review properties of so-called special conformal Killing tensors on a Riemannian manifold $(Q, g)$ and the way they give rise to a Poisson-Nijenhuis structure on the tangent bundle $T Q$. We then address the question of generalizing this concept to a Finsler space, where the metric tensor field comes from a regular Lagrangian function $E$, homogeneous of degree two in the fibre coordinates on $T Q$. It is shown that when a symmetric type $(1,1)$ tensor field $K$ along the tangent bundle projection $\tau: T Q \rightarrow Q$ satisfies a differential condition which is similar to the defining relation of special conformal Killing tensors, there exists a direct recursive scheme again for first integrals of the geodesic spray. Involutivity of such integrals, unfortunately, remains an open problem.
\end{abstract}

Key words: special conformal Killing tensors; Finsler spaces

2000 Mathematics Subject Classification: 37J35; 53C60; 70H06

This paper is based on joint work with Fien Vermeire and Mike Crampin.

\section{Introduction: special conformal Killing tensors}

The work presented here is inspired by the theory and applications of so-called special conformal Killing tensors (or Benenti tensors) on a (pseudo-) Riemannian manifold. The study of possible generalizations to Finsler manifolds should be seen as a first step towards further generalizations to arbitrary metric tensor fields along the tangent bundle projection (coming, for example, from the Hessian of a regular Lagrangian); the aim in the end would be to arrive at a scheme which can cope with integrable or Hamilton-Jacobi separable systems with non-quadratic integrals in involution, as opposed to the subclass of Stäckel systems which is governed by special conformal Killing tensors. We start by reviewing a number of essential features related to such tensors.

Let $g$ be a (pseudo-) Riemannian metric on a manifold $Q$.

Definition 1. A symmetric tensor $J_{i j}$ determines a special conformal Killing tensor (or Benenti tensor) w.r.t. $g$ if its covariant derivatives with respect to the Levi-Civita connection satisfy,

$$
J_{i j \mid k}=\frac{1}{2}\left(\alpha_{i} g_{j k}+\alpha_{j} g_{i k}\right)
$$

for some 1-form $\alpha$.

${ }^{\star}$ This paper is a contribution to the Proceedings of the Workshop on Geometric Aspects of Integrable Systems (July 17-19, 2006, University of Coimbra, Portugal). The full collection is available at http://www.emis.de/journals/SIGMA/Coimbra2006.html 
It follows that

$$
\sum_{i j k} J_{i j \mid k}=\sum_{i j k} \alpha_{i} g_{j k}
$$

i.e. $J$ is a conformal Killing tensor. Further properties (referring to $J$ as $(1,1)$-tensor: $J_{j}^{i}=$ $g^{i k} J_{k j}$ ) are that $J$ is a conformal Killing tensor of gradient type,

$$
\alpha=d f=d(\operatorname{tr} J),
$$

and has vanishing Nijenhuis torsion $N_{J}$, i.e.

$$
[J X, J Y]+J^{2}([X, Y])-J([J X, Y]+[X, J Y])=0 \quad \forall X, Y \in \mathcal{X}(Q) .
$$

Moreover, assuming $J$ is non-singular, its cofactor tensor $A$ :

$$
A J=(\operatorname{det} J) I
$$

is itself a Killing tensor, so that $F=(1 / 2) A^{i j}(q) p_{i} p_{j}$ is a quadratic first integral of the system with Hamiltonian $H=(1 / 2) g^{i j}(q) p_{i} p_{j}$ on $T^{*} Q$.

As said above, metrics admitting a special conformal Killing tensor determine a subclass of Stäckel systems, i.e. orthogonal separable systems in the sense of Hamilton-Jacobi with $n$ quadratic integrals in involution. We briefly sketch how this works, beginning with the integrability structures on $T^{*} Q$ and $T^{*} Q \times \mathbb{R}$, determined by $J$.

On $T^{*} Q$, consider $\widetilde{J}$, the complete lift of $J$, which in coordinates is given by

$$
\widetilde{J}=J_{j}^{i}\left(\frac{\partial}{\partial q^{i}} \otimes d q^{j}+\frac{\partial}{\partial p_{j}} \otimes d p_{i}\right)+p_{k}\left(\frac{\partial J_{i}^{k}}{\partial q^{j}}-\frac{\partial J_{j}^{k}}{\partial q^{i}}\right) \frac{\partial}{\partial p_{i}} \otimes d q^{j} .
$$

If $\omega=d \theta$ denotes the standard symplectic structure and

$$
\omega_{1}=d(J \theta)=d\left(J_{j}^{i} p_{i} d q^{j}\right),
$$

we have that

$$
i_{\widetilde{J}(X)} \omega=i_{X} \omega_{1} \quad \forall X \in \mathcal{X}\left(T^{*} Q\right) .
$$

It follows that $\widetilde{J}$ is symmetric with respect to $\omega$. Moreover, we have $d \omega_{1}=0$ obviously, and $N_{\widetilde{J}}=0$ as a result of $N_{J}=0$. These are the properties which are sufficient to ensure that a special conformal Killing tensor $J$ on $(Q, g)$ determines a Poisson-Nijenhuis structure on $T^{*} Q$ with $\widetilde{J}$ as recursion operator. In addition, we have the interesting relations

$$
d d_{\widetilde{J}} h=d(\operatorname{tr} J) \wedge d h, \quad d d_{\widetilde{J}}(\operatorname{tr} J)=0,
$$

which give rise to an extension of the two compatible Poisson structures to $T^{*} Q \times \mathbb{R}$. Details about all of this can be found in [3].

Observe that the special conformal Killing tensor $J$ plays a kind of double role for the complete integrability of $H=(1 / 2) g^{i j} p_{i} p_{j}$. First of all, we have that $J+s I$ is a special conformal Killing tensor for all $s$, and thus gives rise to a family of corresponding Killing tensors $A(s)$, and in this way to a hierarchy of quadratic integrals. Secondly, the double Poisson structure created by $J$ truly helps to show that such integrals are in involution, in fact with respect to both brackets (although other, more direct techniques for showing involutivity may sometimes be successful as well, see e.g. [5]). Incidentally, the reference just cited also addresses another important aspect of the integrals, namely their functional independence, for which it is shown to be enough that 
the eigenvalues of $J$ are different at one point. To complete the construction of Stäckel systems then, one can proceed as follows. For a function $V$ on $Q$ to be an admissible potential, it is necessary and sufficient that $V$ satisfies

$$
d d_{\widetilde{J}} V=d(\operatorname{tr} J) \wedge d V
$$

Admissibility of $V$ means that

$$
h=\frac{1}{2} g^{i j} p_{i} p_{j}+V
$$

is still separable, and there are then suitable modifications of the first integrals of the kinetic energy part which constitute an amended set of quadratic integrals in involution. To avoid forgetting a number of interesting references concerning details and background about these results, we limit ourselves to citing the excellent review paper [2] and refer to the list of references therein.

\section{A tangent bundle view of special conformal Killing tensors}

We explain some of the results from [4] here; understanding a Lagrangian perspective of what precedes is essential for the generalization to Finsler spaces we have in mind.

Let $(Q, g)$ as before be a pseudo-Riemannian manifold and consider the function

$$
L=\frac{1}{2} g_{i j}(q) u^{i} u^{j} \in C^{\infty}(T Q) .
$$

Then, $T Q$ becomes a symplectic manifold via the Poincaré-Cartan 2-form

$$
\omega_{L}=d \theta_{L}, \quad \text { with } \quad \theta_{L}=S(d L)=\frac{\partial L}{\partial u^{i}} d q^{i},
$$

where $S$ is the canonically defined 'vertical endomorphism' on $T Q$.

Consider then again a type $(1,1)$ tensor field $J$ on $Q$. The following is an interesting result of [4]: it gives a concise, intrinsic characterization of special conformal Killing tensors, directly in their type $(1,1)$ appearance.

Theorem 1. $J$ is a special conformal Killing tensor if and only if it is symmetric with respect to $g$ and satisfies the following relation for some function $f \in C^{\infty}(Q)$ :

$$
\nabla J=\frac{1}{2}\left(\mathbf{T} \otimes d^{H} f-X_{f} \otimes \theta_{L}\right) .
$$

Obviously, we have a bit of work to do here to explain the symbols in this formulation. In principle, this whole formula is about tensor fields along the tangent bundle projection $\tau: T Q \rightarrow Q$, though some of its ingredients so far happen to live on the base manifold $Q$. The operations which are being used rely on the (non-linear) connection associated to a given second-order field $\Gamma$ (here coming from the Euler-Lagrange equations of $L$ ). If we express the coordinate representation of a general second-order field $\Gamma$ as

$$
\Gamma=u^{i} \frac{\partial}{\partial q^{i}}+F^{i}(q, u) \frac{\partial}{\partial u^{i}}
$$

then the basis of horizontal vector fields $H_{i}$ on $T Q$, defining the connection, is given by

$$
H_{i}=\frac{\partial}{\partial q^{i}}-\Gamma_{i}^{j}(q, u) \frac{\partial}{\partial u^{j}}, \quad \text { with } \quad \Gamma_{i}^{j}=-\frac{1}{2} \frac{\partial F^{j}}{\partial u^{i}} .
$$


We have

$$
\left.d^{H} f=H_{i}(f) d q^{i} \quad \text { and } \quad X_{f}\right\lrcorner g=-d^{H} f .
$$

$\mathbf{T}$ is the canonical vector field along $\tau$ (essentially the identity map on $T Q$ ), given by

$$
\mathbf{T}=u^{i} \frac{\partial}{\partial q^{i}}
$$

and $\nabla$, finally, is the dynamical covariant derivative operator. It acts like $\Gamma$ on functions on $T Q$, and is further determined by $\nabla\left(\partial / \partial q^{i}\right)=\Gamma_{i}^{j} \partial / \partial q^{j}$ and by duality on $d q^{j}$. Up to now, however, $f$ and $J$ live on $Q$, so that in fact $d^{H} f=d f$ and, taking into account that we are in a situation where $\Gamma_{i}^{j}=\Gamma_{i k}^{j}(q) u^{k}$, we have $(\nabla J)_{j}^{i}=J_{j \mid k}^{i} u^{k}$. Observe that it easily follows from (1), by taking a trace, that $f=\operatorname{tr} J$.

If $J$ is an arbitrary $(1,1)$ tensor on $Q$, its vertical lift $J^{V}$ defines a kind of alternative almost tangent structure on $T Q$, at least we have $J^{V^{2}}=0$ and $N_{J^{V}}=0$. Therefore, it is a natural construction to let $J^{V}$ take over the role of $S$ for the purpose of defining a second 2-form and the subsequent construction of a type $(1,1)$ tensor $R$ on $T Q$. In other words, natural tangent bundle constructions lead us to define $R$ on $T Q$ by

$$
i_{R(X)} d(S(d L))=i_{X} d\left(J^{V}(d L)\right) \quad \forall X \in \mathcal{X}(T Q),
$$

and if $N_{J}=0$ on $Q$ (as in the case of a special conformal Killing tensor), we have $N_{R}=0$ on $T Q$ as well, so that $R$ becomes the recursion operator of a Poisson-Nijenhuis structure on $T Q$. In

fact, $R$ is precisely the pullback of $\widetilde{J}$ under the Legendre transform associated to the given regular Lagrangian $L$.

\section{Generalization: Finsler manifolds}

Let $(Q, E)$ now be a Finsler space, that is to say, $E: T Q \rightarrow \mathbb{R}$ is the square of a Finsler function $F$, is homogeneous of degree two in the $u^{i}(E(0)=0)$, and $\omega_{E}=d \theta_{E}$ is non-degenerate on the slit tangent bundle $T Q \backslash\{0\}$.

Putting

$$
g_{i j}(q, u)=\frac{\partial^{2} E}{\partial u^{i} \partial u^{j}},
$$

we have a $(0,2)$ tensor field along the tangent bundle projection $\tau$, and consider its canonical spray $\Gamma$ on $T Q \backslash\{0\}$ (i.e. the Euler-Lagrange equations of $E$ ). Note that in view of the homogeneity,

$$
E=\frac{1}{2} g(\mathbf{T}, \mathbf{T})=\frac{1}{2} g_{i j} u^{i} u^{j},
$$

and we also have

$$
\nabla g=0, \quad \nabla \mathbf{T}=0
$$

It is then easy to deduce the following additional properties:

$$
\left.\nabla E=\Gamma(E)=0, \quad d^{H} E=0, \quad \theta_{E}=\mathbf{T}\right\lrcorner g, \quad \nabla \theta_{E}=0 .
$$

It may be worthwhile to make the following preliminary remark to avoid confusion. There is often a lively debate in Finsler geometry about the selection of the best possible linear connection 
to replace the Levi-Civita connection of Riemannian geometry. Most of the time, about four different possibilities are considered (see e.g. [1]), depending on whether one wants the connection to be as metrical as possible, or whether one wants to eliminate as much torsion as possible. We do not need to enter into this debate here, because all we need for deriving our main results in this section is the dynamical covariant derivative operator $\nabla$, as it was specified in the preceding section: it comes from the non-linear connection associated to any second-order differential equation field and acts on the full algebra of tensor fields along the tangent bundle projection $\tau$.

Inspired by the theorem of the preceding section, assume now that we have a type $(1,1)$ tensor $K$ along $\tau$, which is symmetric with respect to $g$, i.e. $g(K X, Y)=g(X, K Y)$, and satisfies a relation of the form

$$
\nabla K=\frac{1}{2}\left(\mathbf{T} \otimes \alpha-X_{\alpha} \otimes \theta_{E}\right),
$$

for some 1-form $\alpha$ along $\tau$, and with $X_{\alpha}$ defined by $\left.X_{\alpha}\right\lrcorner g=-\alpha$. It would be quite natural within this Finsler environment to assume that $K$ is homogeneous of degree zero as well, but as we will show now, such an extra assumption is not even required to arrive at a quite remarkable hierarchy of first integrals for the canonical spray $\Gamma$.

An immediate consequence of (3), which follows from taking a trace, is that

$$
\alpha(\mathbf{T})=\nabla \operatorname{tr} K
$$

As a result, if we put

$$
h_{0}=k_{0}=E, \quad k_{1}=\frac{1}{2} g(\mathbf{T}, K \mathbf{T}),
$$

it is easy to show that also

$$
h_{1}=k_{1}-E \operatorname{tr} K
$$

is a first integral. This is the start for the hierarchy of first integrals, for which we found the following explicit recursive scheme. Define

$$
k_{j}=\frac{1}{2} g\left(\mathbf{T}, K^{j} \mathbf{T}\right), \quad \text { and } \quad a_{j}=\frac{1}{j} \operatorname{tr} K^{j}, \quad j=1,2, \ldots
$$

Lemma 1. From the fundamental assumption (3) on $K$, it follows that

$$
\begin{aligned}
& \nabla a_{j}=\alpha\left(K^{j} \mathbf{T}\right), \\
& \nabla k_{j}=\sum_{i=1}^{j}\left(\nabla a_{i}\right) k_{j-i} .
\end{aligned}
$$

Proof. It is easy to show by induction that (3) implies that

$$
\nabla K^{j+1}=\frac{1}{2} \sum_{i=0}^{j}\left(K^{i} \alpha \otimes K^{j-i} \mathbf{T}-K^{i} \theta_{E} \otimes K^{j-i} X_{\alpha}\right) .
$$

Taking a trace, (4) immediately follows. Moreover, since

$$
\nabla k_{j}=\frac{1}{2} g\left(\mathbf{T}, \nabla K^{j}(\mathbf{T})\right),
$$

a direct computation, using the formula for $\nabla K^{j}$ just derived, leads to (5). 
Next, we introduce auxiliary functions $\phi_{k}$, defined recursively by

$$
\phi_{1}=0, \quad \phi_{k}=\frac{1}{2} \sum_{i=1}^{k-1} a_{i} a_{k-i}-\frac{1}{k} \sum_{i=2}^{k-1}(k-i) \phi_{i} a_{k-i}, \quad k \geq 2 .
$$

Lemma 2. We have $\nabla \phi_{j}=\sum_{l=1}^{j-1} a_{l} \nabla a_{j-l}-\sum_{l=2}^{j-1} \phi_{l} \nabla a_{j-l}, \quad j \geq 2$.

Proof. From $\phi_{2}=(1 / 2) a_{1}^{2}$, we get $\nabla \phi_{2}=a_{1} \nabla a_{1}$ and the property is clearly true for $j=2$. Assume it is valid for all $j$ up to $k-1$ and now act with $\nabla$ on $\phi_{k}$ as defined by (6). We get

$$
\begin{aligned}
\nabla \phi_{k}= & \sum_{i=1}^{k-1} a_{i} \nabla a_{k-i}-\frac{1}{k} \sum_{i=2}^{k-1}(k-i) \phi_{i} \nabla a_{k-i}-\frac{1}{k} \sum_{i=2}^{k-1}(k-i) a_{k-i} \sum_{l=1}^{i-1} a_{l} \nabla a_{i-l} \\
& +\frac{1}{k} \sum_{i=2}^{k-1}(k-i) a_{k-i} \sum_{l=2}^{i-1} \phi_{l} \nabla a_{i-l} .
\end{aligned}
$$

In order to collect coefficients of $\nabla a_{j}$, we put $j=k-i$ in the second term on the right and $j=i-l$ in the last two terms (and adjust the summations). Moreover, we subsequently interchange the two summations in those last two terms and split off one term to have the common upper bound $k-3$ for $j$. The result reads

$$
\begin{aligned}
\nabla \phi_{k}= & \sum_{i=1}^{k-1} a_{i} \nabla a_{k-i}-\frac{1}{k} \sum_{j=1}^{k-2} j \phi_{k-j} \nabla a_{j} \\
& -\frac{1}{k} \sum_{j=1}^{k-3} \nabla a_{j}\left(\sum_{i=j+1}^{k-1}(k-i) a_{k-i} a_{i-j}-\sum_{i=j+2}^{k-1}(k-i) a_{k-i} \phi_{i-j}\right)-\frac{1}{k} a_{1}^{2} \nabla a_{k-2} .
\end{aligned}
$$

In the terms between brackets, we now change $i-j$ to $l$, thus getting

$$
\left(\sum_{l=1}^{k-j-1}(k-j-l) a_{k-j-l} a_{l}-\sum_{l=2}^{k-j-1}(k-j-l) a_{k-j-l} \phi_{l}\right)
$$

which upon closer inspection can be seen to be equal to $(k-j) \phi_{k-j}$. It is now a matter of splitting off the term for $j=k-2$ in the second sum on the right also, to see a few cancellations presenting themselves, which then readily produce the desired result.

Finally, put $b_{k}=\phi_{k}-a_{k}$ and define

$$
h_{l}=k_{l}+\sum_{i=1}^{l} b_{i} k_{l-i}, \quad l \geq 1 \text {. }
$$

Theorem 2. Let $K$ be a type $(1,1)$ tensor along $\tau: T Q \rightarrow Q$, which is symmetric with respect to the Finsler metric $g$ and satisfies condition (3) for some $\alpha$. Then the functions $h_{l}$ are first integrals of the geodesic spray of the Finsler metric $g$, for all $l$.

Proof. From Lemma 1 we have, using also $\nabla k_{0}=0$,

$$
\nabla h_{l}=\sum_{i=1}^{l}\left(\nabla \phi_{i}\right) k_{l-i}+\sum_{i=1}^{l-1} b_{i} \sum_{j=1}^{l-i}\left(\nabla a_{j}\right) k_{l-i-j} .
$$


Putting $m=i+j$ in the second term and interchanging the double summation, this becomes

$$
\nabla h_{l}=\sum_{i=2}^{l}\left(\left(\nabla \phi_{i}\right)+\sum_{m=1}^{i-1} b_{m}\left(\nabla a_{i-m}\right)\right) k_{l-i}+\left(\nabla \phi_{1}\right) k_{l-1} .
$$

This is clearly zero in view of $\phi_{1}=0$ and Lemma 2 .

It is interesting to verify that one can rewrite the recursive formula for integrals in the following alternative way:

$$
h_{l}=\frac{1}{2} g\left(\mathbf{T}, B_{l} \mathbf{T}\right) \quad \text { with } \quad B_{l}=b_{l} I+B_{l-1} K, \quad B_{0}=I .
$$

Moreover, the $b_{l}$ turn out to be the coefficients of the characteristic polynomial of $K$ : indeed, if $n$ is the dimension of $Q$, one can show that

$$
\operatorname{det}(\lambda I-K)=\sum_{i=0}^{n} b_{i} \lambda^{n-i} \quad\left(b_{0}=1\right),
$$

which means that in particular $b_{n}=(-1)^{n} \operatorname{det} K$.

Obviously, this whole construction applies in particular to the Riemannian case with the assumption that $K$ is a special conformal Killing tensor on $Q$; in fact, the expression (7) looks like a direct generalization of a result of Benenti (see the first theorem in section 7 of [2]).

The parallel with the Riemannian case goes further, in the sense that, if $K$ is assumed to be non-singular, there is again a first integral associated to the cofactor tensor. Observe, by the way, that the quadratic integral $F=(1 / 2) A^{i j}(q) p_{i} p_{j}$ referred to in section 1 for the Riemannian case, can be written in a tangent bundle set-up in the coordinate free format $F=(1 / 2) g(A \mathbf{T}, \mathbf{T})$, and this statement happens to extend to the Finslerian situation.

Theorem 3. Let $g$ be a Finsler metric, and $K$ a symmetric $(1,1)$ tensor satisfying condition (3). Then, if $K$ is non-singular and $A$ is its cofactor tensor, the function $g(A \mathbf{T}, \mathbf{T})$ is a first integral of the geodesic spray.

Proof. We have that $\nabla A=\nabla(\operatorname{det} K) K^{-1}-(\operatorname{det} K) K^{-1} \nabla K K^{-1}$ and it is a general property for derivation of degree zero such as $\nabla$ that $\nabla \log (\operatorname{det} K)=\operatorname{tr}\left(K^{-1} \nabla K\right)$. It follows that

$$
\begin{aligned}
\nabla(g(A \mathbf{T}, \mathbf{T})) & =g(\nabla A(\mathbf{T}) T) \\
& =(\operatorname{det} K)\left(\operatorname{tr}\left(K^{-1} \nabla K\right) g\left(K^{-1} \mathbf{T}, \mathbf{T}\right)-g\left(\nabla K K^{-1}(\mathbf{T}), K^{-1}(\mathbf{T})\right)\right) .
\end{aligned}
$$

From the assumption (3) on $K$, it readily follows that $\operatorname{tr}\left(K^{-1} \nabla K\right)=\alpha\left(K^{-1}(\mathbf{T})\right)$ and

$$
g\left(\nabla K K^{-1}(\mathbf{T}), K^{-1}(\mathbf{T})\right)=\alpha\left(K^{-1}(\mathbf{T})\right) g\left(\mathbf{T}, K^{-1} \mathbf{T}\right) .
$$

Hence, $\nabla(g(A \mathbf{T}, \mathbf{T}))=0$.

The hierarchy of first integrals derived above can now be established via the cofactor technique as well. Indeed, if $K$ satisfies the fundamental condition (3), then so does $K+s I$ for all real values $s$. Its cofactor $A(s)=\sum_{j=0}^{n-1} A_{j+1} s^{j}$ therefore also gives rise to a hierarchy of first integrals.

In fact, we have

$$
(K+s I) A(s)=\sum_{j=0}^{n-1} K A_{j+1} s^{j}+\sum_{j=1}^{n} A_{j} s^{j}
$$


On the other hand, using (8) we get

$$
\operatorname{det}(K+s I)=(-1)^{n} \operatorname{det}((-s) I-K)=\sum_{i=0}^{n}(-1)^{i} b_{i} s^{n-i} .
$$

It follows that

$$
A_{n}=I, \quad \text { and } \quad K A_{1}=(-1)^{n} b_{n} I
$$

which shows that $A_{1}=A$, while the other coefficients in the expansion of $A(s)$ have to satisfy the recursive relation

$$
K A_{j+1}+A_{j}=(-1)^{n-j} b_{n-j} I, \quad j=1, \ldots, n-1 .
$$

Going down this scheme from top to bottom, it is easy to see that $A_{n-j}=(-1)^{j} B_{j}$, which shows (see (7)) that the coefficients in the expansion of $A(s)$ determine the same first integrals $h_{l}$ up to sign. Notice that, in particular, $A=A_{1}=(-1)^{n-1} B_{n-1}$, from which it follows that

$$
B_{n}=B_{n-1} K+b_{n} I=(-1)^{n-1} A K+(-1)^{n}(\operatorname{det} K) I=0 .
$$

One can verify also that $b_{n+1} \equiv 0$. Hence, the sequence of first integrals actually terminates with $h_{n}=0$ and the cofactor tensor $A$ of $K$ determines the last non-zero integral $h_{n-1}$ in the hierarchy.

\section{The problem of involutivity}

We have not succeeded in coming anywhere near to proving that the integrals we obtained in the previous section would be in involution with respect to the Poisson bracket associated to the symplectic form $d \omega_{E}$. One would expect to have better chances if one could use the tensor $K$ for constructing a recursion tensor $R$ of a Poisson-Nijenhuis structure on $T Q$, just as in the Riemannian case. Observe that the construction (2) of $R$ in the Riemannian case in fact makes sense under much more general circumstances. Indeed, the vertical lift $J^{V}$ remains well defined if we replace the basic tensor $J$ on $Q$ by a tensor $J$ along the projection $\tau$, and the function $L$ can be taken to be any regular Lagrangian on a tangent bundle $T Q$. Such an $R$ then is determined, through its action on vertical and horizontal lifts of vector fields $X$ along $\tau$, by formulas of the form (see [6])

$$
\begin{aligned}
& R\left(X^{V}\right)=(\bar{K} X)^{V}, \\
& R\left(X^{H}\right)=(K X)^{H}+(U X)^{V},
\end{aligned}
$$

where $\bar{K}$ is the transpose of $K$ (with respect to $g$ ) and $K$ and $U$ are defined by

$$
\begin{aligned}
& g(K X, Y)=\mathrm{D}_{Y}^{V}\left(J \theta_{L}\right)(X), \\
& g(U X, Y)=d^{H}\left(J \theta_{L}\right)(X, Y) .
\end{aligned}
$$

We refer to [6] for details about the vertical covariant derivative operator $\mathrm{D}^{V}$ and the horizontal exterior derivative $d^{H}$ in these determining equations.

If we think of such a construction in the Finslerian case under consideration, with $L=E$, it is natural to assume that $J$ is homogeneous of degree zero and then $K$ has the same property. If we then assume that this $K$ satisfies the assumptions we needed for the hierarchy of first integrals, namely $K=\bar{K}$ and the condition (3), then, unfortunately, there is still no guarantee 
that $N_{R}=0$. Indeed, in the Finslerian case, although the generically five different components of $N_{R}$ reduce to two, and these considerably simplify further in view of the above assumptions on $K$, in the end we run out of luck and they do not automatically vanish! Note in passing that the vertical and horizontal covariant derivative operators which appear in the intrinsic characterization of the tensors $K$ and $U$ above are associated, for the Finslerian case, to the Berwald connection. It is very unlikely, however, that a different selection of horizontal subspaces to describe these tensors (i.e. the choice of one of the other linear connections, familiar in Finsler geometry) might have any effect on the problem of involutivity of the integrals. It further remains an open problem whether there are different, more direct ways of obtaining some form of involutivity. Also, the issue of functional independence of the integrals, as treated in [5] for the Riemannian case, has not been addressed.

\section{Acknowledgement}

This work has been partially supported by the European Union through the FP6 Marie Curie RTN ENIGMA (Contract number MRTN-CT-2004-5652).

\section{References}

[1] Bao D., Chern S.-S., Shen Z., An introduction to Riemann-Finsler geometry, Graduate Texts in Mathematics, Vol. 200, Springer-Verlag, New York, 2000.

[2] Benenti S., Special symmetric two-tensors, equivalent dynamical systems, cofactor and bi-cofactor systems, Acta Appl. Math. 87 (2005), 33-91.

[3] Crampin M., Sarlet W., Thompson G., Bi-differential calculi, bi-Hamiltonian systems and conformal Killing tensors, J. Phys. A: Math. Gen. 33 (2000), 8755-8770.

[4] Sarlet W., Vermeire F., A class of Poisson-Nijenhuis structures on a tangent bundle, J. Phys. A: Math. Gen. 37 (2004), 6319-6336, math.DG/0402076.

[5] Topalov P., Matveev V.S., Geodesic equivalence via integrability, Geom. Dedic. 96 (2003), 91-115.

[6] Vermeire F., Sarlet W., Crampin M., A class of recursion operators on a tangent bundle, J. Phys. A: Math. Gen. 39 (2006), 7319-7340. 